\title{
Aromatase expression in low-grade endometrial stromal sarcomas: an immunohistochemical study
}

\author{
Olaf Reich $^{1}$ and Sigrid Regauer ${ }^{2}$ \\ ${ }^{1}$ Department of Obstetrics and Gynecology and ${ }^{2}$ Department of Pathology, University of Graz, Graz, Austria
}

\begin{abstract}
Aromatase expression has been described in stromal cells of endometriosis, adenomyosis and endometrial cancer. We analyzed aromatase expression in a series of 23 low-grade endometrial stromal sarcomas. Archival formalin-fixed and paraffin-embedded material was analyzed with immunohistochemistry. Aromatase expression was evaluated with a monoclonal and a polyclonal antibody using the peroxidase-antiperoxidase method. A score was calculated based on the percentage of positive tumor cells and the staining intensity. Aromatase was seen in $19(83 \%)$ of 23 tumors with monoclonal antibody and $20(87 \%)$ of 23 tumors with polyclonal antibody. Aromatase expression using the monoclonal antibody was scored as high in five (22\%), moderate in nine $(39 \%)$ and low in five $(22 \%)$ tumors. Four $(17 \%)$ low-grade endometrial stromal sarcomas did not stain for aromatase. Aromatase expression with the polyclonal antibody was scored as high in seven (31\%), moderate in four $(17 \%)$ and low in nine $(39 \%)$ tumors. Three $(13 \%)$ low-grade endometrial stromal sarcomas did not stain for aromatase. Little or no aromatase expression tended to correlate with stage I disease, while higher scores were more frequently associated with advanced disease. Our results demonstrate that most low-grade endometrial stromal sarcomas express aromatase. The staining pattern, however, is heterogeneous. The high percentage of aromatase positivity in low-grade endometrial stromal sarcomas may have implications in the management of these tumors and offer new treatment modalities such as hormonal therapy with aromatase inhibitors.

Modern Pathology (2004) 17, 104-108, advance online publication, 19 November 2003; doi:10.1038/modpathol.3800031
\end{abstract}

Keywords: endometrium; stromal sarcoma; aromatase; treatment

Low-grade endometrial stromal sarcomas are rare, accounting for about $0.2 \%$ of all genital tract malignancies. ${ }^{1}$ They occur predominantly in premenopausal women. ${ }^{2}$ Little is known of their etiology or natural history. Low-grade endometrial stromal sarcomas are hormone sensitive and estrogen can act as a growth stimulus. ${ }^{3}$ Most women with low-grade endometrial stromal sarcomas undergo bilateral salpingo-oophorectomy as part of primary treatment. However, estrogen can also be produced by extraovarian sources and likely by tumor cells themselves. ${ }^{4-7}$ This extraovarian estrogen production depends on conversion of circulating androgen to estrogen via the aromatase enzyme complex. The aromatase enzyme complex consists of the CYP19 gene product aromatase cytochrome $P 450$ and a flavoprotein NADPH-cytochrome $P 450$ reductase

Correspondence: O Reich, Department of Obstetrics and Gynecology, University of Graz, Auenbruggerplatz 14, A-8036 Graz, Austria.

E-mail: olaf.reich@kfunigraz.ac.at

Received 16 June 2003; revised 12 August 2003; accepted 18 August 2003; published online 19 November 2003 and is located in the endoplasmic reticulum of estrogen-producing cells. ${ }^{4}$

Tseng et $a l^{8}$ reported concentrations of aromatase in eight uterine sarcomas, which were within the range of activity found in normal proliferative endometrium or above normal levels. These authors used cytosol-based biochemical examinations and their observations have not been confirmed in larger studies. Immunohistochemical quantification of aromatase has not been reported for low-grade endometrial stromal sarcomas. In the present study, we analyzed aromatase expression in a series of 23 low-grade endometrial stromal sarcomas using monoclonal and polyclonal antibodies.

\section{Materials and methods}

Histologic sections of 23 low-grade endometrial stromal sarcomas treated between 1983 and 2002 were retrieved from the archives and re-evaluated by two pathologists (SR and OR). All tumors were composed of cells resembling normal endometrial stromal cells invading the myometrium and vascular spaces. Mitoses were fewer than 10/10 high-power 
fields $(\mathrm{HPF}=$ objective $\times 40)$ and necrosis was absent. The tumors were staged according to the International Federation of Gynecology and Obstetrics (FIGO) classification for endometrial carcinoma. $^{9}$

For immunohistochemical analysis, one or two paraffin blocks with representative portions of the tumors were selected and $2-\mu \mathrm{m}$ thick sections were stained with monoclonal (1:100 dilution, SM1671P, Acris, Germany) and polyclonal antibody (1:1000 dilution). The anti-aromatase polyclonal antibody was the rabbit immunoglobulin fraction of an antiserum prepared against human placental aromatase as previously decribed $^{10}$ and was kindly provided by Dr N Harada, Fujita Health University Hospital of (Toyaoake, Japan). The peroxidaseantiperoxidase method was used to detect the antigens. Aromatase expression was evaluated by two pathologists (SR and OR). Placental tissue served as positive controls. A score was calculated based of the percentage of positive tumor cells $(<10 \%=1 ; \quad 11-50 \%=2 ; \quad 51-80 \%=3 ;>81 \%=4)$ multiplied by the staining intensity (negative $=0$; weak $=1$; moderate $=2$; strong $=3$ ). A minimum of $1 \%$ of tumor cells had to be positive to be called positive staining. A final score of 0 was classified as negative, $1-3$ as low; 4-6 as medium and 8-12 as high.

The mean age of the patients at surgery was 45 years (range 25-79) and only four patients were postmenopausal. A total of 15 patients had surgical stage I, seven had stage III, and one stage IV disease. In total, 22 patients underwent total abdominal hysterectomy with bilateral salpingo-oophorectomy. One patient (pt.) had only exploratory laparotomy (pt. 5). Seven patients (pts. 1-4, 7, 8, 23) with stage III disease additionally had an omentectomy and one patient (pt. 7) underwent pelvic lymphadenectomy. Six patients (pts. 1, 3, 4, 7, 9, 23) recieved adjuvant chemotherapy, one patient (pt. 2) adjuvant radiation, and 16 patients no further treatment. Seven patients were lost to follow-up and the median follow-up of the remaining 16 patients was 8.2 (1-16) years. Five patients (pts. 3, 4, 7, 9, 17) developed pelvic recurrences and one patient (pt. 5) developed lung and bone metastases. Two patients (pts. 2 and 5) died of disease and one patient (pt. 18) of unrelated causes.

\section{Results}

Aromatase was seen in 19 (83\%) of 23 tumors with monoclonal antibody and $20(87 \%)$ of 23 tumors with polyclonal antibody. Aromatase expression using the monoclonal antibody was scored as high in five $(22 \%)$, moderate in nine $(39 \%)$ and low in five $(22 \%)$ tumors (Figures 1 and 2). Four (17\%) low-grade endometrial stromal sarcomas did not stain for aromatase. Aromatase expression with the polyclonal antibody was scored as high in seven

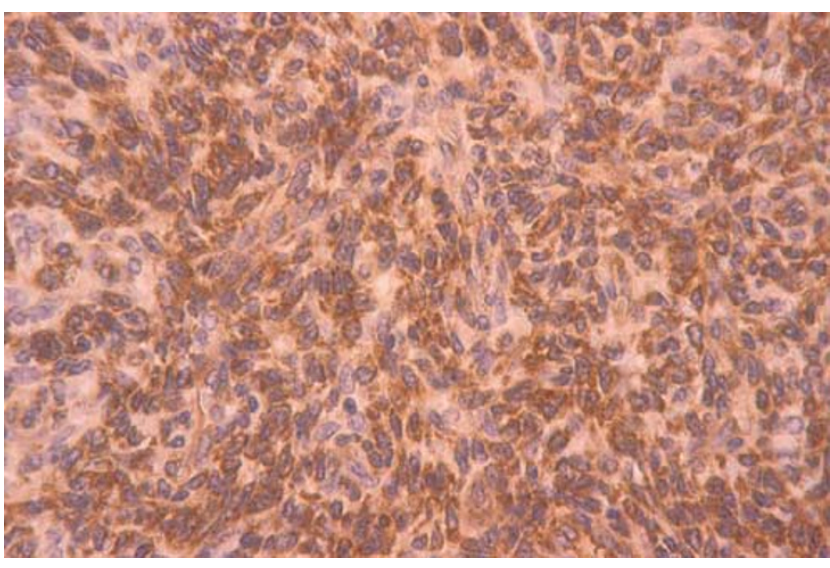

Figure 1 Low-grade endometrial stromal sarcoma with overall high aromatase expression with homogeneous strong diffuse cytoplasmic staining in the absence of nuclear staining (pt. 4, score 12). Antiaromatase, monoclonal, peroxidase-antiperoxidase, original magnification $\times 200$.

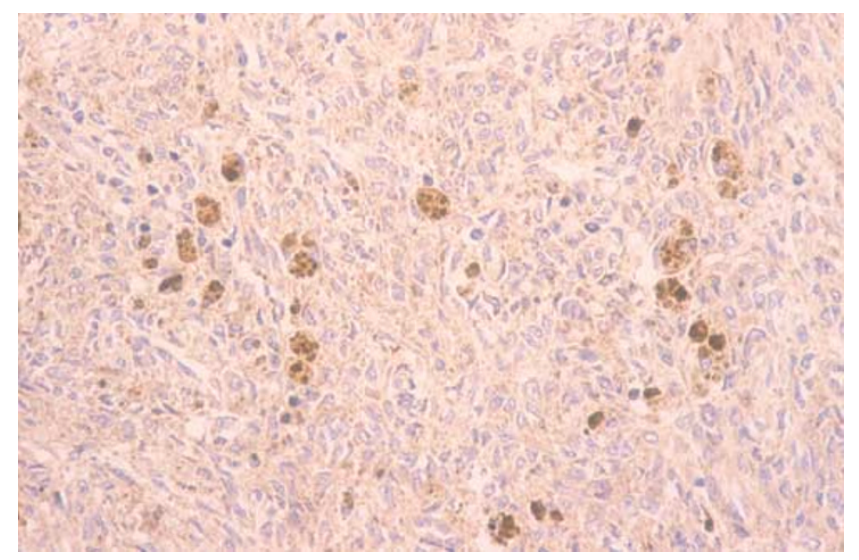

Figure 2 Low-grade endometrial stromal sarcoma with overall low aromatase expression. Only individual cells or small groups of cells show strong granular cytoplasmic staining, while the majority of cells do not stain specifically (pt. 14, score 3). Antiaromatase, monoclonal, peroxidase-antiperoxidase, original magnification $\times 200$.

$(31 \%)$, moderate in four $(17 \%)$ and low in nine (39\%) tumors. Three (13\%) low-grade endometrial stromal sarcomas did not stain for aromatase (Table 1).

Low-grade endometrial stromal sarcomas with a high score for aromatase were uniformly positive throughout the entire tumor with strong and diffuse cytoplasmic staining (Figure 1). Low-grade endometrial stromal sarcomas with a moderate score showed variable staining. In low-grade endometrial stromal sarcomas with a low score, the majority of the tumor cells were negative. The staining intensity of positive cells ranged from weakly to strongly positive with either a diffuse or a granular cytoplasmic staining pattern (Figure 2).

Despite the small study numbers, low-grade endometrial stromal sarcomas with no or low aromatase expression tended to correlate with stage I disease, while higher scores were more frequently 
Table 1 Expression of aromatase in 23 patients with low-grade endometrial stromal sarcomas

\begin{tabular}{|c|c|c|c|c|c|c|c|c|c|c|c|c|}
\hline \multirow[t]{2}{*}{$\begin{array}{l}\text { Pt. } \\
\text { no. }\end{array}$} & \multirow[t]{2}{*}{$\begin{array}{c}\text { Age } \\
\text { (years) }\end{array}$} & \multirow{2}{*}{$\begin{array}{c}\text { Stage } \\
\text { (FIGO) at } \\
\text { presentation }\end{array}$} & \multirow[t]{2}{*}{$\begin{array}{l}\text { Adjuvant } \\
\text { therapy }\end{array}$} & \multirow[t]{2}{*}{$\begin{array}{l}\text { Clinical } \\
\text { follow-up }\end{array}$} & \multicolumn{3}{|c|}{$\begin{array}{c}\text { Anti-aromatase } \\
\text { monoclonal }\end{array}$} & \multirow{2}{*}{$\begin{array}{l}\text { Interpretation } \\
\text { (overall } \\
\text { expression) }\end{array}$} & \multicolumn{3}{|c|}{$\begin{array}{c}\text { Anti-aromatase } \\
\text { polyclonal }\end{array}$} & \multirow{2}{*}{$\begin{array}{l}\text { Interpretation } \\
\text { (overall } \\
\text { expression) }\end{array}$} \\
\hline & & & & & Cells & SI & Score & & Cells & $S I$ & Score & \\
\hline 1 & 50 & III & CT & Lost & 3 & 3 & 9 & High & 4 & 2 & 8 & High \\
\hline 2 & 65 & III & RT & DOD & 4 & 3 & 12 & High & 3 & 3 & 9 & High \\
\hline 3 & 44 & III & CT & AWD & 3 & 2 & 6 & Medium & 2 & 3 & 6 & Medium \\
\hline 4 & 35 & III & CT & AWD & 4 & 3 & 12 & High & 4 & 3 & 12 & High \\
\hline 5 & 35 & IV & - & DOD & 3 & 2 & 6 & Medium & 4 & 2 & 8 & High \\
\hline 6 & 46 & I & - & NED & 1 & 2 & 2 & Low & 1 & 3 & 3 & Low \\
\hline 7 & 35 & III & CT & AWD & 4 & 2 & 8 & High & 3 & 3 & 9 & High \\
\hline 8 & 38 & III & - & NED & 2 & 1 & 2 & Low & 1 & 3 & 3 & Low \\
\hline 9 & 35 & I & CT & AWD & 1 & 2 & 2 & Low & 1 & 1 & 1 & Low \\
\hline 10 & 47 & I & - & NED & 0 & 0 & 0 & Negative & 0 & 0 & 0 & Negative \\
\hline 11 & 74 & I & - & Lost & 0 & 0 & 0 & Negative & 0 & 0 & 0 & Negative \\
\hline 12 & 34 & I & - & NED & 0 & 0 & 0 & Negative & 0 & 0 & 0 & Negative \\
\hline 13 & 38 & I & - & NED & 3 & 3 & 9 & High & 3 & 3 & 9 & High \\
\hline 14 & 50 & I & - & NED & 1 & 3 & 3 & Low & 1 & 3 & 3 & Low \\
\hline 15 & 45 & I & - & NED & 2 & 3 & 6 & Medium & 1 & 3 & 3 & Low \\
\hline 16 & 25 & I & - & Lost & 1 & 3 & 3 & Low & 1 & 2 & 2 & Low \\
\hline 17 & 35 & I & - & AWD & 2 & 2 & 4 & Medium & 1 & 2 & 2 & Low \\
\hline 18 & 79 & I & - & DWD & 2 & 2 & 4 & Medium & 2 & 2 & 4 & Medium \\
\hline 19 & 39 & I & - & Lost & 2 & 3 & 6 & Medium & 2 & 2 & 4 & Medium \\
\hline 20 & 34 & I & - & Lost & 2 & 2 & 4 & Medium & 3 & 3 & 9 & High \\
\hline 21 & 72 & I & - & Lost & 3 & 2 & 6 & Medium & 3 & 1 & 3 & Low \\
\hline 22 & 44 & I & - & NED & 2 & 2 & 4 & Medium & 2 & 2 & 4 & Medium \\
\hline 23 & 42 & III & CT & Lost & 0 & 0 & 0 & Negative & 1 & 1 & 1 & Low \\
\hline
\end{tabular}

CT, chemotherapy; RT, radiotherapy; NED, no evidence of disease; AWD, alive with recurrent disease; DWD, dead without disease; DOD, dead of disease; Lost, lost to follow-up. Cells: Percentage of positive tumor cells $(<10 \%=1 ; 11-50 \%=2 ; 51-80 \%=3 ;>81 \%=4)$. SI: Staining intensity (negative $=0$; weak $=1$; moderate $=2$; strong $=3$ ). Score: Cells $\times$ SI, Interpretation: Score 1-3 as low; 4-6 as medium and 8-12 as high.

associated with advanced stages (Table 1). In the aromatase-positive group, 10 of 12 patients with stage I disease had low or medium scores, only two patients (pts. 13 and 20) had a high score. With the exception of patient 23, all aromatase-negative lowgrade endometrial stromal sarcomas corresponded to stage I disease (pts. 10-12). Of the five patients with recurrent disease (pts. 3, 4, 7, 9, 17) one had a low score (pt. 9), two had a medium score (pts. 3 and 17), and two had a high score (pts. 4 and 7). The two women who died of disease (pts. 2 and 5) had a score of 8 (high) and 12 (high), respectively (monoclonal or polyclonal antibody, Table 1).

\section{Discussion}

Aromatase expression has been described in stromal cells of endometriosis, adenomyosis and endometrial cancer, ${ }^{4,11-13}$ but not in stromal cells of eutopic endometrium or endometrial hyperplasia. ${ }^{13}$ This is the first immunohistochemical study of aromatase expression in low-grade endometrial stromal sarcomas. We found that most tumors expressed aromatase (Table 1 and Figures 1 and 2). There was a wide agreement among the staining results obtained with monoclonal and polyclonal antibody, and the differences were limited to one category (Table 1). Staining was heterogeneous. The small number of patients in our study precludes speculation about associations between clinical behavior and this heterogeneous aromatase expression, but low scores appeared to be more common in stage I disease, while higher scores were more frequently seen in patients with advanced diseases. The two women who died of disease showed high aromatase expression (Table 1).

Aromatase is a key enzyme in estrogen biosynthesis, and estrogen seems to be a promoting factor in low-grade endometrial stromal sarcomas. ${ }^{3}$ Lowgrade endometrial stromal sarcomas have been reported in women with exogenous or endogenous hyperestrogenism. ${ }^{14-16}$ Among women with stage I low-grade endometrial stromal sarcomas, a higher recurrence rate is found in patients with residual ovarian tissue when compared with those treated with bilateral oophorectomy. ${ }^{14}$ Similarly, regression in size of metastases have been reported after bilateral oophorectomy ${ }^{17}$ and radiation-induced castration. ${ }^{18} \mathrm{~A}$ lower incidence of recurrence and longer disease-free survivals after bilateral oophorectomy have also been reported..$^{19}$

Recurrences develop in one-third to one-half of patients with low-grade endometrial stromal sarcomas and can appear as long as 30 years after primary therapy. ${ }^{20-22}$ Little is known about the reasons for such a high recurrence rate after primary surgery. A possible explanation could be a growth stimulus by estrogen on residual tumor cells. After oophorectomy, estrogen can be produced by peripheral 
tissues such as the skin, muscle and fat. ${ }^{4}$ Also, aromatase-positive tumor cells may produce estrogen by converting androgens. Immunohistochemical demonstration of aromatase expression may aid in identifying tumor cells utilizing this pathway of estrogen production in low-grade endometrial stromal sarcomas. The heterogeneous staining pattern indicates that only a subpopulation of tumor cells in low-grade endometrial stromal sarcomas is capable of estrogen production.

Analysis of intratumoral aromatase expression in low-grade endometrial stromal sarcomas may shed light on the development and biological behavior of these tumors and may have implications for clinical mangement. Aromatase inhibitors are now widely used in treatment of estrogen-dependent breast cancer. ${ }^{14,23,24}$ Aromatase inhibitors reduce estrogen levels by inhibiting estrogen synthesis in both tumor tissues and peripheral sites, and as a consequence they inhibit the proliferation of the tumor. Aromatase inhibitors may also influence apoptosis. ${ }^{24}$ Aromatase inhibitors are being studied for other hormone-dependent benign and malignant tumors. ${ }^{24,25}$ The demonstration of aromatase expression in low-grade endometrial stromal sarcomas may be a rationale for a study of aromatase inhibitors in the treatment of women with lowgrade endometrial stromal sarcomas. Spano et $a l^{26}$ have reported initial results of such adjuvant hormonal therapy. In their series, two patients with metastatic low-grade endometrial stromal sarcomas had a complete response after treatment with an aromatase inhibitor.

In conclusion, our results demonstrate that most low-grade endometrial stromal sarcomas express aromatase. Aromatase expression has been shown to be associated with tumor growth, maintenance and progression and may also play a role in the high recurrence rate of low-grade endometrial stromal sarcomas. Aromatase inhibitors appear to represent an intriguing novel treatment modality of low-grade endometrial stromal sarcomas to reduce growth stimulatory effects of estrogens in this entity.

\section{Acknowledgements}

We thank Dr N Harada of The First Department of Biochemistry (Fujita Health University Hospital, Toyaoake, Japan) for kindly providing his antiaromatase antibody. The authors are grateful to Dr F Nogales, University of Granada, Spain for contributing a case and Dr K Tamussino for editing the manuscript.

\section{References}

1 Koss LG, Spiro RH, Brunschwig A. Endometrial stromal sarcoma. Surg Gynecol Obstet 1965;121: 531-537.
2 Evans HL. Endometrial stromal sarcoma and poorly differentiated endometrial sarcoma. Cancer 1982;50: 2170-2182.

3 Reich O, Regauer S, Urdl W, et al. Expression of estrogen and progesterone receptors in low-grade endometrial stromal sarcomas. Br J Cancer 2000;82: 1030-1034.

4 Sasano H, Harada N. Intratumoral aromatase in human breast, endometrial, and ovarian malignancies. Endocr Rev 1998;19:593-607.

5 Miller WR, Mullen P, Telford J, et al. Clinical importance of intratumoral aromatase. Breast Cancer Res Treat 1998;49:27-32.

6 Sumitani H, Shozu M, Segawa T, et al. In situ estrogen synthesized by aromatase P450 in uterine leiomyoma cells promotes cell growth probably via an autocrine/ intracrine mechanism. Endocrinology 2000;141: 3852-3861.

7 Zhang Z, Yamashita H, Toyama T, et al. Semiquantitative immunohistochemical analysis of aromatase expression in ductal carcinoma in situ of the breast. Breast Cancer Res Treat 2002;74:47-53.

8 Tseng L, Tseng JK, Mann WJ, et al. Endocrine aspects of human uterine sarcoma: a preliminary study. Am J Obstet Gynecol 1986;155:95-101.

9 Benedet JL, Pocorelli S. Staging Classification and Clinical Practice Guidelines of Gynecologic Cancers. Elsevier: Oxford, 2000.

10 Harada N. Novel properties of human placental aromatase as cytochrome P-450: purification and characterization of a unique form of aromatase. J Biochem (Tokyo) 1988;103:106-113.

11 Yang S, Fang Z, Suzuki T, et al. Regulation of aromatase $P 450$ expression in endometrioitic and endometrial stroma cells by CCAAT/enhancer binding proteins (C/EBPs): decreased C/EBP $\beta$ in endometriosis is associated with overexpression of aromatase. J Clin Endocrinol Metab 2002;87:2336-2345.

12 Balun SE, Yang S, Fang Z, et al. Role of aromatase in endometrial disease. J Steroid Biochem Mol Biol 2001;79:19-25.

13 Watanabe K, Sasano H, Harada N, et al. Aromatase in human endometrial carcinoma and hyperplasia. Immunohistochemical, in situ hybridisation, and biochemical studies. Am J Pathol 1995;146:491-500.

14 Genazzani AR, Gadducci A, Gambaccini M. Controversial issues in climacteric medicine II: hormone replacement therapy and cancer. Maturitas 2001;40: 117-130.

15 Horowitz K, Rutherford T, Schwartz PE. Hormone replacement therapy in women with sarcomas. CME J Gynecol Oncol 1996;1:23-29.

16 Press MF, Scully RE. Endometrial 'sarcomas' complicating ovarian thecoma, polycystic ovarian disease and estrogen therapy. Gynecol Oncol 1985;21:135-154.

17 Baggish MS, Woodruff JD. Uterine stromatosis: clinicopathologic features and hormone dependency. Obstet Gynecol 1972;40:487-498.

18 Gloor E, Schneyder P, Cikes M, et al. Endolymphatic stromal myosis. Surgical and hormonal treatment of extensive abdominal recurrence 20 years after hysterectomy. Cancer 1982;50:1888-1893.

19 Krieger PD, Gusberg SB. Endolymphatic stromal myosis-a grade I endometrial sarcoma. Gynecol Oncol 1973;1:299-313.

20 Chang KL, Crabtree GS, Lim-Tan SK, et al. Primary uterine endometrial stromal neoplasms. A clinico- 
pathologic study of 117 cases. Am J Surg Pathol 1990;14:415-438.

21 Clement BC, Young RH. Tumors and Tumor-like Lesions of the Uterine Corpus and Cervix. Churchill Livingstone: New York, 1993.

22 Styron SL, Burke TW, Linville WK. Low-grade endometrial stromal sarcoma recurring over three decades. Gynecol Oncol 1989;35:275-278.

23 Brueggemeier RW. Aromatase, aromatase inhibitors, and breast cancer. Am J Ther 2001;8:333-344.
24 Sasano H, Sato S, Ito K, et al. Effects of aromatase inhibitors on the pathology of human breast, endometrial and ovarian carcinoma. Endocr Relat Cancer 1999;6:197-204.

25 Noble LS, Simpson ER, Johns A, et al. Aromatase expression in endometriosis. J Clin Endocrinol Metab 1996;81:174-179.

26 Spano JP, Soria JC, Kambouchner M, et al. Long term survival of patients given hormonal therapy for metastatic endometrial stromal sarcoma. Med Oncol 2003;20:87-93. 